\title{
Concurrent Chemoradiotherapy for Malignant Glioma: Dead End or Highway?
}

\author{
René-Olivier Mirimanoff \\ Department of Radiation Oncology, CHUV, University Hospital Medical Center, Lausanne, Switzerland
}

Malignant or high-grade glioma (HGG) are the most common and devastating of all adult brain tumours. Their incidence has steadily increased over the years, especially in patients in their 5 th decade and over [1]. Surgery and radiotherapy (RT) have been the mainstay of treatment for more than 40 years [2], while before the landmark EORTC/NCI-C trial on temozolomide (TMZ) [3], chemotherapy - which was mainly based on nitrosoureas - yielded only a marginal improvement in survival, if any [4]. Amongst many drugs, paclitaxel was also tested in conjunction with RT in HGG, based on in vitro data demonstrating that the drug caused a block in G2/M, a more radiosensitive phase of the cell cycle [5]. In this issue of ONKOLOGIE, Montemor and colleagues present a randomized study conducted between 1998 and 2002, in which 61 patients with either glioblastoma (GBM) or anaplastic astrocytoma (AA) were randomized to receive radiotherapy with or without weekly paclitaxel [6]. The results indicate that the combined regimen was well tolerated, but independently of tumour grade (GBM or AA), there was apparently no statistical difference between the two treatment groups. The authors conclude that radiochemotherapy with paclitaxel is safe but that there is no increase in overall survival. They formulate the hypothesis that the difference between the in vitro and in vivo (or clinical) radiosensitising effect of paclitaxel in malignant glioma is likely to be due to the lack of permeability of the blood-brain barrier to this drug. Later in their discussion, and alluding to the results of the EORTC/NCI-C TMZ trial, they state that the survival benefit with $\mathrm{TMZ}$ being 2.5 months, other treatments should be investigated.

On one hand, Montemor and colleagues should be commended for having tested paclitaxel and RT in a randomized trial, given the fact that the literature on HGG is replete with phase II trials testing various drugs and RT with no control arm. These studies are of course hard to interpret because especially in GBM, pre-therapeutic prognostic factors have a more powerful impact on survival than most treatments [7]. On the other hand, the study presented here has important limitations and several of the investigator's statements are quite disputable. To start with, and strictly speaking, the authors are not in a position to conclude that 'there was no increase in overall survival with weekly paclitaxel'. The study was not powered sufficiently to demonstrate a difference, and even less powered to demonstrate the absence of difference between the two treatment arms due to the very small number of patients in each subset. Nevertheless, and assuming that there was indeed no effect of paclitaxel with RT, one can speculate at length about the reasons why the combination did not work. For example the blood-brain-barrier issue, including the authors' proposal to modulate it, may have little relevance in HGG, especially with RT which can in itself alter the bloodbrain-barrier. There are most likely several other 'culprits' for the lack of efficacy of the drug or its combination with RT, such as 'classical' resistance factors [8] (i.e. tumour proliferation, $\mathrm{pH}$, hypoxia etc.) or more recently described ones $[9,10]$ (i.e. RT-induced invasion via integrins, MGMT expression, EGFR, VEGF, PDGF expression etc.), all of which can play a critical role in HGG resistance. In order to better identify the various causes of failure of chemotherapy and combined chemoradiotherapy, and in the hope of overcoming these, it is now essential to systematically perform translational studies in parallel with any new clinical trial in GBM. In this regard the demonstration by the EORTC/NCI-C TMZ trial that the $M G M T$ (methylguanine-DNA-methyltransferase) status has a prognostic and predictive value [11] will enable us to define new treatment strategies depending on this particular tumour state. Another explanation of the discrepancy between the in vitro and in vivo data may be the lack of daily RT exposure to paclitaxel simply because the drug had to be given weekly. Thus, one cannot really speak here of a true concurrent chemoradiotherapy scheme, contrary to the EORTC/NCI-C

\begin{tabular}{|c|c|}
\hline KARGER & (c) 2008 S. Karger GmbH, Freiburg \\
\hline $\begin{array}{l}\text { Fax +4976145207 14 } \\
\text { E-mail Information@Karger.de } \\
\text { www.karger.com }\end{array}$ & $\begin{array}{l}\text { Accessible online at: } \\
\text { www.karger.com/onk }\end{array}$ \\
\hline
\end{tabular}


RT-TMZ protocol where the drug is indeed given every day during radiation, which may prove much more efficient than a weekly schedule. In this respect, there are some solid preclinical data to support a positive interaction between TMZ and radiation when given concurrently: 1) TMZ and RT inhibit GBM cells in a dose-dependent fashion [12];2) like paclitaxel, TMZ induces an arrest in G2M [13]; 3) TMZ inhibits radiation-induced invasion via inhibition of integrins [14]; 4) TMZ increases radiation-induced DNA double strand breaks and cell death when the drug is given concomitantly with radiotherapy [15]; 5) continuous administration of low-dose TMZ depletes MGMT [16]. The EORTC/NCI-C trial of concomitant and adjuvant TMZ and RT for newly diagnosed GBM has unequivocally demonstrated for the first time in decades that the right drug, when given the proper way in conjunction with RT, improved overall survival not only statistically significantly but also in a clinically meaningful way. Here, I would like to comment on the statement of Montemor and colleagues that 'the median survival benefit with temozolomide was 2.5 months [and] other treatments should be investigated'. Does it mean that TMZ should be replaced by other drugs or that other drugs should be added to TMZ? Firstly: in the EORTC/NCI-C trial, the survival curves (TMZ+RT vs. RT) start to diverge only after about 6 months. So assessing treatment efficacy using median survival, which is done too often inappropriately in oncology, is much less relevant here than assessing the survival at 2 years and beyond. Secondly: the updated results of the trial showed that at 2,3,4 and 5 years, the overall survival in the combined treatment group was 27,16 , 12 and $10 \%$, respectively, indicating a modest but definitive proportion of patients who are alive well beyond what could be expected with post-operative RT alone [17]. Some subgroups of patients with a favourable prognosis (i.e. RPA III and those with methylated $M G M T$ ) had even a fourfold increase of survival at 3, 4 and 5 years, respectively with TMZ and RT. So this means reasonable hope for at least a subgroup of patients. Even if, and unfortunately a majority of patients will not be cured, the association of TMZ and RT is considered now as a new treatment standard, against which any new therapy should be compared. New strategies should include the addition of novel agents to the RT-TMZ backbone, and actually there are several trials testing so-called targeted therapies (i.e. cilengitide, temsirolimus, cetuximab, bevacuzimab, etc.), most of which are combined with RT and TMZ [10]. In a good number of these studies, there are convincing preclinical data to support their use with RT and TMZ, and in addition translational research is being carried out in parallel. It is hoped that these new treatment strategies will lead to improved outcome in HGG. Dead-end or highway? The answer could be both, depending on how badly or how well future studies will be conceived, carried out and analyzed. The entrance to the highway may not be so far away.

\section{References}

1 Hess KR, Broglio K, Bondy ML: Adult glioma incidence trends in the United States 1977-2000. Cancer 2004;101:2293-2299.

2 Laperriere N, Zuraw L, Cairncross G: The Cancer Care Ontario Practice Guidelines Initiative NeuroOncology Disease Site Group. Radiotherapy for newly diagnosed malignant glioma in adults: a systematic review. Radiother Oncol 2002;64:259-73.

-3 Stupp R, Mason WP, van den Bent M, Weller M, Fisher B, Taphoorn MJ et al.: Radiotherapy plus concomitant and adjuvant temozolomide for glioblastoma. N Engl J Med 2005;352:987-96.

$\checkmark 4$ Stewart LA: Chemotherapy in adult high-grade glioma: a systematic review and meta-analysis of individual patient data from 12 randomised trials. Lancet 2002;359:1011-18.

$\checkmark 5$ Geard CR, Jones JM, Schiff PB: Taxol and radiation. J Natl Cancer Inst Monogr 1993;15:89-94.

6 Montemor JP, Peria FM, Monti CR, Petrilli LS, Colli BO, Carlotti CG: Concurrent chemoradiotherapy with weekly paclitaxel in malignant cerebral glioma treatment. Onkologie 2008;31:435-439.
7 Mirimanoff RO, Gorlia T, Mason W, van den Bent MJ, Kortmann RD, Fisher B et al.: Radiotherapy and temozolomide for newly diagnosed glioblastoma: recursive partitioning analysis of the EORTC 26981/22981-NCIC CE3 phase III randomized trial. J Clin Oncol 2006;24:2563-69.

8 Kortmann RD, Jeremic B, Weller M, Plasswilm ML, Bamberg M: Chemoradiotherapy of malignant glioma in adults - clinical experiences. Strahlenther Onkol 2003;179:219-232.

9 Behin A, Hoang-Xuan K, Carpentier AF, Delattre JY: Primary brain tumours in adults. Lancet 2003;361:323-331.

10 Stupp R, Hegi ME, Gilbert MR, Chakravarti A: Chemotherapy in malignant glioma: standard of care and future directions. J Clin Oncol 2007;25: 4127-36.

11 Hegi ME, Diserens AC, Gorlia T, Hamou MF, de Tribolet N, Weller M et al.: MGMT gene silencing and benefit from temozolomide in glioblastoma. $\mathrm{N}$ Engl J Med 2005:352:997-1003.

12 Wedge SR, Porteous JK, Glaser MG: In vitro evaluation of temozolomide combined with X-irradiation. Anticancer Drugs 1997;8:92-7.
3 Hirose Y, Berger MS, Pieper RO: P53 effects both the duration of G2/M arrest and the fate of temozolomide-treated human glioblastoma cells. Cancer Res 2001;61:1957-63.

14 Wick W, Wick A, Schulz JB, Dichgans J, Rodenmann HP, Weller M: Prevention of irradiation-induced glioma cell invasion by temozolomide involves caspase 3 activity and cleavage of focal-adhesion kinase. Cancer Res 2002;62:1915-9.

15 Chakravarti A, Erkkinen MG, Nestler U, Stupp R, Mehta M, Aldape K, et al.: Temozolomide-mediated radiation enhancement in glioblastoma: a report on underlying mechanisms. Clin Cancer Res 2006; 12:4738-46.

16 Tolcher AW, Gerson SL, Denis L, Geyer C, Hammond LA Patnaik A, et al.: Marked inactivation of 06-alkylguanine DANN alkyl-transferase with protracted temozolomide schedules. Br J Cancer 2003; 88:1004-11.

17 Mirimanoff RO, Mason W, van den Bent M, Kortmann RD, Taphoorn M, Brandes A, et al.: Is longterm survival in glioblastoma possible? Updated results of the EORTC/NCIC phase III randomized trial on radiotherapy (RT) and concomitant and adjuvant temozolomide versus RT alone. Int J Radiat Oncol Biol Phys 2007;69 (3S):2. 